\title{
Substitution of wheat flour with whole-grain spelt flour in cakes and its influence on consumer acceptance
}

\author{
C. Kong ${ }^{1}$, C. Soh ${ }^{2}$ and G. M. O’Brien ${ }^{1}$ \\ ${ }^{1}$ School of Agriculture, Food and Rural Development, Newcastle University (Singapore Campus) and ${ }^{2}$ Nanyang \\ Polytechnic, School of Chemical and Life Sciences
}

In typical bakery products, wheat flour is the principal ingredient ${ }^{(1)}$, and wheat gluten has been associated with negative health consequences in individuals with gluten-related disorders ${ }^{(2)}$. Wholegrain spelt flour (WSF) appears to offer a suitable alternative to wheat for some gluten-sensitive individuals. In order to be accepted by consumers, any 'healthy alternative' version of a food product must be of comparable sensory quality to the standard product ${ }^{(3)}$. To date, new product-development (NPD) efforts involving WSF have mainly been focused on breads, with little NPD work in the area of other baked goods such as cakes ${ }^{(1)}$. The aim of this study was to develop WSF versions of sponge cake and butter cake - both popular in Singapore -, with similar baking quality and product acceptability to those of the standard wheat-based versions. In a series of controlled bakery trials, baking performance (specific volume: $\mathrm{cm}^{3} / \mathrm{g}$ ) of WSF- and standard wheat-based versions was compared (seed-displacement method). During a five-day post-baking period, crumb firmness ( $\mathrm{g}$ ) and springiness (\%) were compared (TX - XT Plusä Texture Analyzer). All assays were performed in triplicate. Analysis of the resulting data indicated an absence of significant difference $(p>0.05)$ between WGS- and standard wheat-based sponge cakes and butter cakes, in terms of specific volume, firmness and springiness (Mann-Whitney/Kruskal-Wallis tests used).

In consumer testing, the perceptions of 50 mainly Singaporean consumers (aged 19-37 years) regarding the wheat- and WGS-based sponge cake and butter cake products were assessed using a 9-point hedonic scale ${ }^{(4)}$. At the $5 \%$ significance level, the wheat-based butter cake scored significantly better than the WGS-based butter cake in terms of overall appearance, overall aroma and texturesoftness, while the wheat-based sponge cake outscored the WGS version in terms of overall appearance and overall aroma. With the remaining attributes ('flavour' and 'overall acceptance', and additionally 'texture-softness' in the case of the sponge cake), no significant differences $(p>0.05)$ were noted between the wheat-based and WGS-based cakes (Mann-Whitney U test used).

The results of the study demonstrate the potential of WGS as a potential substitute for wheat flour in some cake-type products. Although no significant differences were found in terms of specific volume, firmness and springiness between the WGS- and the standard wheat-based cakes, nonetheless the consumer-derived data indicate something of a "preference gap" in favour of the standard wheat-based products. If WGS-based cake products are to be fully successful, it appears that NPD efforts will be required to focus on improving sensory quality.

1. Abdel-Aal ESM. \& Rabalski I (2008) Food Chem 111, 150-156.

2. Catassi C, Bai JC, Bonaz B et al. (2013) Nutrients 5, 3839-3853.

3. Fogliano V \& Vitaglione P (2005) Mol Nutr Food Res 49, 256-262.

4. Resurrección AVA (2008) Consumer sensory testing for product development. In: Brody A.L. and Lord J.B (eds.) Developing New Food Products for a Changing Marketplace (2nd ed) CRC Press, Boca Raton, USA. 